\title{
Chromosomal Loci Associated with Antibiotic Hypersensitivity in Pulmonary Isolates of Pseudomonas aeruginosa
}

\author{
By JANET A. M. FYFE* AND JOHN R. W. GOVAN \\ Department of Bacteriology, University of Edinburgh Medical School, Teviot Place, \\ Edinburgh EH8 9AG, UK
}

(Received 18 October 1983; revised 23 November 1983)

\begin{abstract}
492a and 492c were two strains of Pseudomonas aeruginosa isolated from the sputum of a patient with cystic fibrosis. The strains were closely related but expressed different antibiograms. $492 \mathrm{c}$ was hypersensitive (10-100 times more sensitive than 492a) to the $\beta$-lactam antibiotics carbenicillin, methicillin, flucloxacillin, mecillinam and cefuroxime and the non- $\beta$-lactam, nalidixic acid. $492 \mathrm{c}$ also showed enhanced sensitivity (4-8 times more sensitive than $492 \mathrm{a}$ ) to chloramphenicol, trimethoprim and novobiocin. 492a and PAO8 expressed similar levels of antibiotic resistance, except for trimethoprim, to which 492a was five times more sensitive than PAO8. Two genes associated with antibiotic hypersensitivity were mapped in the $30 \mathrm{~min}$ region of the chromosome, by means of R68.45-mediated plate matings between a Leu ${ }^{-}$mutant of $492 \mathrm{c}$ and PAO8, followed by transductional analysis using phage F116L. The first of these genes, blsAl, was closely linked to nalB, and in a PAO background, was associated with hypersensitivity to the $\beta$-lactams and a moderate increase in sensitivity to chloramphenicol, trimethoprim, nalidixic acid and novobiocin. A further increase in sensitivity to the latter three antibiotics was associated with the second gene, $\operatorname{tps} A 1$, which mapped between ser-3 and his $V$. This gene could also be transferred to PAO from 492a, thus 492c could have arisen from 492a in vivo following a single chromosomal mutation at the $b l s A$ locus. Isolation of a $b l s A$ mutant of PAO969 provided further evidence for this theory.
\end{abstract}

\section{INTRODUCTION}

One of the characteristics most commonly associated with Pseudomonas aeruginosa is an intrinsic resistance to antimicrobial agents, in particular, the $\beta$-lactam antibiotics. Indeed, this property is generally held responsible for the emergence of $P$. aeruginos $a$ in the last three decades as a clinically important opportunist pathogen.

The development of carbenicillin, the first semi-synthetic penicillin with significant antipseudomonas activity, was a significant advance in chemotherapy. However, the minimal inhibitory concentration (MTC) of carbenicillin for $P$. aeruginosa is generally $25-50 \mu \mathrm{g} \mathrm{ml}^{-1}$ (Knudsen et al., 1967), which is beyond the levels attainable in soft tissues, e.g. the lung (Marks et al., 1971).

May \& Ingold (1972) reported that strains of $P$. aeruginosa isolated from sputum were often considerably more sensitive to carbenicillin than isolates from other sources. In a study involving 111 sputum isolates from patients with cystic fibrosis (CF), chronic bronchitis and bronchiectasis, they found that $35 \%$ of strains were sensitive to $6 \mu \mathrm{g}$ carbenicillin $\mathrm{ml}^{-1}$ and some had MICs as low as $0.7 \mu \mathrm{g} \mathrm{ml}^{-1}$.

\footnotetext{
Abbreviations: CEF, cefuroxime; CF, cystic fibrosis; DSTA, Diagnostic Sensitivity Test Agar; NA, nutrient agar; NB, nutrient broth; TP, trimethoprim.
} 
Berche et al. (1979) reported a study on antibiotic susceptibilities of mucoid and non-mucoid strains of $P$. aeruginosa. They compared 47 mucoid with 71 non-mucoid isolates on the basis of sensitivities to 18 antibiotics and concluded that both groups could be divided into two distinct classes on this basis. Class A contained strains significantly more resistant to antibiotics such as the aminoglycosides and tetracycline, than those strains allocated to Class B.

While studying the association of $P$. aeruginosa with chronic respiratory infection in CF patients, we have also reported the isolation of strains unusually sensitive (hypersensitive) to a range of antibiotics including the $\beta$-lactams carbenicillin, azlocillin, methicillin and also to trimethoprim (Irvin et al., 1981). The carbenicillin MICs of these strains were $<1 \mu \mathrm{g} \mathrm{ml}^{-1}$ and thus comparable with those reported by May \& Ingold (1972). In addition, we reported that hypersensitive and 'normal' isolates could be obtained from the same sputum specimen and that these strains were invariably of the same pyocin type, indicating a close relationship.

Recently, there has been an increasing interest in the genetic basis of virulence in $P$. aeruginosa and various potential virulence factors have been mapped using the genetically characterized strain, PAO. For example, several chromosomal genes involved in exotoxin A production have been mapped following isolation of toxin-deficient PAO mutants, and the use of classical genetic techniques (Gray \& Vasil, 1981 a; Hanne et al., 1983). Similarly, a chromosomal gene controlling phospholipase $\mathrm{C}$ production has been identified (Gray \& Vasil, $1981 b$ ). Both of these potential virulence factors are normally expressed in PAO, but others may be expressed only following a mutation, for example, alginate production leading to a mucoid phenotype which is so common in $P$. aeruginosa isolated from the sputa of CF patients. Mucoid mutants of PAO have been isolated in vitro and two chromosomal loci involved in the control of alginate synthesis have been mapped (Govan \& Fyfe, 1978; Fyfe \& Govan, 1980; J. A. M. Fyfe \& J. R. W. Govan, unpublished).

A third approach to the genetic analysis of virulence involves the transfer of the relevant gene(s) from a clinical isolate into a PAO background. This approach is applicable to both chromosomally-encoded and plasmid-encoded characteristics, and has been used in the genetic analysis of extracellular protease production (Wretlind et al., 1983) and transposable antibiotic resistance (Sinclair \& Holloway, 1982).

In the course of studying the intrinsic antibiotic resistance of $P$. aeruginosa strain PAO, various loci associated with this characteristic have been mapped on the chromosome (Mills \& Holloway, 1976; Okii et al., 1983; Matsumoto \& Terawaki, 1982). Functional aspects of the $P$. aeruginosa outer membrane have been examined using an antibiotic 'supersusceptible' mutant of strain 799 (Zimmerman, 1980; Angus et al., 1982; Darveau \& Hancock, 1983). However, genetic analysis of this strain proved unsuccessful (Angus et al., 1982).

The genetic basis of antibiotic hypersensitivity as expressed in clinical isolates of $P$. aeruginosa has not, to our knowledge, been reported. May \& Ingold (1972) postulated that the normal resistance to carbenicillin might be plasmid-encoded, and that this plasmid may sometimes be lost during growth in the respiratory tract. However, no evidence was presented to support this hypothesis. The present paper describes the genetic mapping of antibiotic hypersensitivity in the clinical isolate 492c (Irvin et al., 1981) by means of inter-strain crosses with suitably marked PAO recipients, followed by transductional analysis using phage F116L (Krishnapillai, 1971). A preliminary report of this work has been presented (Fyfe \& Govan, 1983).

\section{METHODS}

Bacteria and bacteriophages. The bacterial strains used in this study are shown in Table 1. Donor strains carrying the plasmid R68.45 were constructed according to Haas \& Holloway (1976). Phage F116L (Krishnapillai, 1971) was used for transduction and F116c for strain construction.

Media and cultural conditions. Nutrient broth (NB), nutrient agar (NA), and minimal agar have been described previously (Fyfe \& Govan, 1980). Amino acid supplements were added at a concentration of $1 \mathrm{~mm}$. The antibiotics used were carbenicillin (Pyopen; Beecham), methicillin (Celbenin; Beecham), benzyl penicillin (Crystapen; Glaxo), ampicillin (Penbritin; Beecham), flucloxacillin (Floxapen; Beecham), mecillinam (Leo Pharmaceuticals), cefuroxime (Zinacef; Glaxo), cefoxitin (Mefoxin; MSD), tetracycline (Glaxo), trimethoprim lactate (Wellcome), nalidixic acid (Winthrop), novobiocin (Sigma), rifampicin (Roche), streptomycin (Glaxo), gentamicin (Roussel), 
Table 1. Strains of $P$. aeruginosa used in this study

Strain

PAOI

$\mathrm{PAO} 2$

PAO4

PAO8

PAO222

PAO969

PAO6002

PAO6006

PAO6511

PAO6524

PAO6526

GMA037

$492 \mathrm{a}$

492a Leu $^{-}$

$492 \mathrm{c}$

$492 \mathrm{c} \mathrm{Leu}^{-}$

PAJ 1

PAJ2

PAJ3

PAJ5

PAJ6
Genotype/Description*

Prototroph, $\mathrm{chl}-2$

ser-3

arg-47 pyrB52

met-28 ilv-202 str-1

met-28 trp-6 lys-12 his-4 ilv-226 pro-82

proC 130

met-9011 amiE200 nalB4

proCI30 nalB9

proC 130 blsA2

proC 130 nalB4 derivative constructed using F116c

Pro $^{+}$transductant of PAO6511 constructed using F116c

hisV5037

Clinical isolate, mucoid, prototrophic, tpsA

Leucine auxotroph isolated from 492 a following EMS mutagenesis

Clinical isolate, mucoid, prototrophic, blsAl tpsAl

Leucine auxotroph isolated from $492 \mathrm{c}$ following EMS mutagenesis

Prototrophic, tpsAl blsAI recombinant from cross $492 \mathrm{c}$

$\mathrm{Leu}^{-} \mathrm{R} 68.45 \times \mathrm{PAO} 8$

Prototrophic, blsAl recombinant from cross $492 \mathrm{c} \mathrm{Leu}^{-} \mathrm{R} 68.45$ $\times$ PAO8

Prototrophic, tpsA1 recombinant from cross $492 \mathrm{cLu}^{-} \mathrm{R} 68.45$ $\times$ PAO8

met-28 tps A1, recombinant from cross $492 \mathrm{c} \mathrm{Leu}^{-} \mathrm{R} 68.45 \times \mathrm{PAO} 8$

ilv-202 tpsA1, transductant of PAJ5 using F116c grown on PAO8 $\mathrm{met}^{+}$
Reference

Holloway (1969)

Isaac \& Holloway (1968)

B. W. Holloway collection

Isaac \& Holloway (1968)

Haas \& Holloway (1976)

Rella \& Haas (1982)

Rella \& Haas (1982)

Rella \& Haas (1982)

This paper

This paper

This paper

Mee \& Lee (1967)

Irvin et al. (1981)

This paper

Irvin et al. (1981)

This paper

This paper

This paper

This paper

This paper

This paper

* Genotype symbols are the same as those used for $E$. coli; bls $A$ designates sensitivity to $\beta$-lactam antibiotics, $\operatorname{tps} A$ sensitivity to trimethoprim, ami acetamide utilization and str resistance to streptomycin.

tobramycin (Lilley), and kanamycin (Bristol Laboratories). All antibiotics were incorporated in Diagnostic Sensitivity Test Agar (DSTA; Oxoid) at the appropriate concentration.

Broth cultures were grown in McCartney bottles on an orbital incubator (Gallenkamp) at 140 r.p.m., and all cultures were incubated at $37^{\circ} \mathrm{C}$, unless stated otherwise.

Isolation of mutants. Auxotrophic mutants were isolated after EMS mutagenesis (Watson \& Holloway, 1976) and carbenicillin enrichment (Watson \& Holloway, 1978).

Carbenicillin-hypersensitive mutants were isolated following EMS mutagenesis and screening of colonies by replica plating onto DSTA plates containing $5 \mu \mathrm{g}$ carbenicillin $\mathrm{ml}^{-1}$.

Antibiotic sensitivity testing. Exponential phase NB cultures were diluted in physiological saline to a concentration of $10^{5}$ organisms $\mathrm{ml}^{-1}$. A multiple inoculator (Mast) was used to dispense samples (containing $10^{2}-10^{3}$ cells) onto DSTA plates appropriately supplemented with the antibiotics. The MIC was read as the lowest concentration of antibiotic that caused complete growth inhibition after $18 \mathrm{~h}$ incubation at $37^{\circ} \mathrm{C}$.

Plate matings. The procedure used was that described by Stanisich \& Holloway (1972). Prior to inter-strain matings, NB cultures of the recipient strains were incubated for $18 \mathrm{~h}$ at $43^{\circ} \mathrm{C}$ (Rolfe \& Holloway, 1966).

Transductions. These were performed according to the method of Krishnapillai (1971).

\section{RESULTS}

\section{Antibiotic sensitivities}

It has previously been reported that $492 \mathrm{a}$ and $492 \mathrm{c}$ differ in their sensitivities to carbenicillin, azlocillin, piperacillin, ticarcillin, methicillin, tetracycline and trimethoprim (Irvin et al., 1981).

We determined the MICs of an extended range of antibiotics for $492 \mathrm{a}$ and $492 \mathrm{c}$, and the results are shown in Table 2. Strain PAO8 was also included to confirm that the MICs obtained for this strain were comparable with 492a and not the hypersensitive 492c. However, 492a was five times more sensitive to trimethoprim than PAO8. Table 2 shows that $492 \mathrm{c}$ is generally hypersensitive to the $\beta$-lactam antibiotics with the following exceptions: benzyl penicillin, 


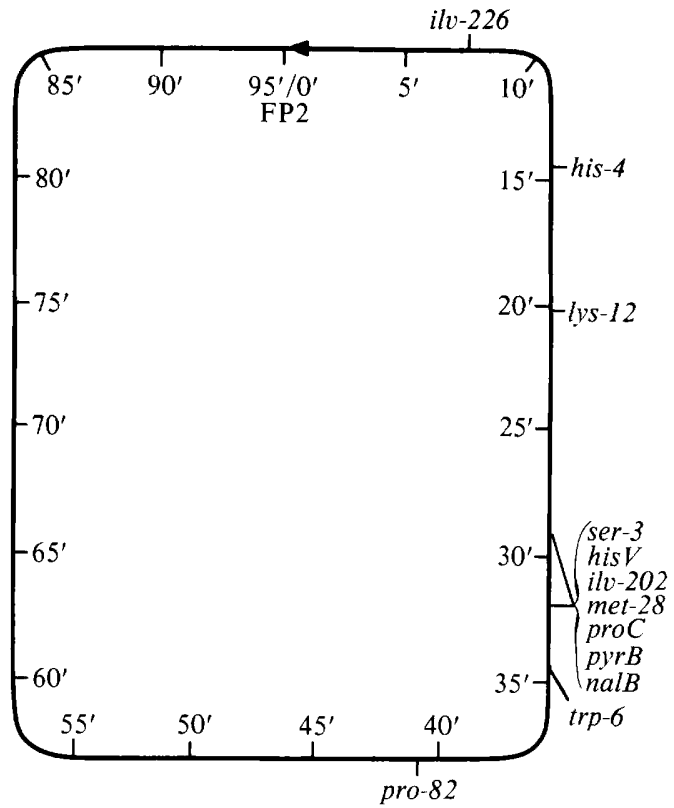

Fig. 1. Genetic map of $P$. aeruginosa showing markers relevant to this study. Based on Holloway \& Crockett (1982).

Table 2. Sensitivities of P. aeruginosa strains $492 a, 492 c$ and PAO8 to antibiotics

\begin{tabular}{|c|c|c|c|}
\hline \multirow[b]{2}{*}{ Antibiotic } & \multicolumn{3}{|c|}{$\mathrm{MIC}\left(\mu \mathrm{g} \mathrm{ml}^{-1}\right)$} \\
\hline & $492 \mathrm{a}$ & $492 c$ & PAO8 \\
\hline Carbenicillin & 80 & $0 \cdot 6$ & 40 \\
\hline Methicillin & $>500$ & 10 & 500 \\
\hline Benzyl penicillin & 500 & 500 & $>1000$ \\
\hline Ampicillin & 100 & 100 & 200 \\
\hline Flucloxacillin & $>500$ & 50 & $>500$ \\
\hline Mecillinam & 500 & 10 & 500 \\
\hline Cefuroxime & $>500$ & 10 & 400 \\
\hline Cefoxitin & 400 & 400 & 500 \\
\hline Cephaloridine & 500 & 500 & $>1000$ \\
\hline Chloramphenicol & 40 & 10 & 60 \\
\hline Tetracycline & 10 & 6 & 10 \\
\hline Trimethoprim & 40 & 5 & 200 \\
\hline Nalidixic acid & 60 & 2 & 80 \\
\hline Novobiocin & $>500$ & 100 & 500 \\
\hline Rifampicin & 40 & 40 & 20 \\
\hline Streptomycin & 40 & 40 & $>500^{*}$ \\
\hline Gentamicin & $0 \cdot 6$ & $0 \cdot 6$ & $0 \cdot 6$ \\
\hline Tobramycin & $0 \cdot 4$ & $0 \cdot 4$ & 0.2 \\
\hline Kanamycin & 60 & 60 & 60 \\
\hline
\end{tabular}

* PAO8 is resistant to streptomycin due to the $s t r-1$ locus.

ampicillin, cefoxitin and cephaloridine. Increased sensitivity to trimethoprim was confirmed for $492 \mathrm{c}$, and also noted for nalidixic acid and novobiocin. No differences between 492a and $492 \mathrm{c}$ were observed for rifampicin, streptomycin, gentamicin, tobramycin or kanamycin.

Transfer of antibiotic hypersensitivity from $492 \mathrm{c}$ to $P A O$

Figure 1 is a chromosome map of $P$. aeruginosa PAO showing the locations of markers relevant to this study. A donor derivative of $492 \mathrm{c}$ was prepared by isolating a Leu ${ }^{-}$mutant, and 
Table 3. Results of recombinant analysis following a plate mating between $492 \mathrm{c} \mathrm{\textrm {Leu } ^ { - }}$ (R68.45) and $P A O 8$

\begin{tabular}{|c|c|c|c|c|}
\hline \multirow[b]{2}{*}{ Selected marker } & \multicolumn{4}{|c|}{ Percentage co-inheritance of unselected character } \\
\hline & $\mathrm{CEF}^{\mathrm{*} *}$ & $\mathrm{TP}+$ & Met $^{+}$ & $\mathrm{Ilv}^{+}$ \\
\hline met $-28^{+}$ & 80 & 82 & - & 77 \\
\hline$i l v-202$ & 75 & 88 & 87 & - \\
\hline
\end{tabular}

transferring R68.45 into this strain. Plate matings were performed between $492 \mathrm{c} \mathrm{Leu}^{-}$(R68.45) and the multiple-marker recipient PAO222. Selection was made for each of the auxotrophic markers and 100 recombinants for each marker were scored for co-inheritance of cefuroxime sensitivity (on DSTA plus $100 \mu \mathrm{g}$ cefuroxime $\mathrm{ml}^{-1}$ ). Increased sensitivity to cefuroxime rather than carbenicillin was chosen initially to avoid any problems associated with the inheritance of R68.45 by some of the recombinants (R68.45 codes for a type IIIa $\beta$-lactamase which is active against carbenicillin but not cefuroxime). Recombinant analysis revealed that $56 \%$ of the met$28^{+}$colonies were sensitive to $100 \mu \mathrm{g}$ cefuroxime $\mathrm{ml}^{-1}$ and likewise $20 \%$ of the $\operatorname{trp}-6^{+}$colonies. Less than $1 \%$ of the recombinants for the other markers, i.e. ilv-226, his-4, lys-12 and pro- 82 had co-inherited this characteristic.

In order to map the locus associated with increased cefuroxime sensitivity more precisely, a plate mating was performed between 492c Leu (R68.45) and PAO8 (met-28 ilv-202). Selection was made for both auxotrophic markers and recombinants scored for both cefuroxime and trimethoprim sensitivity (on DSTA plus $100 \mu \mathrm{g}$ trimethoprim $\mathrm{ml}^{-1}$ ). The results of this recombinant analysis are shown in Table 3 , and indicate that cefuroxime sensitivity $(C E F)$ and trimethoprim sensitivity $\left(\mathrm{TP}^{\mathrm{s}}\right)$ do not always cosegregate. All $\mathrm{CEF}^{\mathrm{s}}$ recombinants were $\mathrm{TP}^{\mathrm{s}}$, but a percentage of the $\mathrm{TP}^{\mathrm{s}}$ colonies were $\mathrm{CEF}^{\mathrm{r}}$.

The MICs for representatives of each recombinant class, i.e. $C E F^{s} T^{s}$, $C^{2} F^{r} T^{s}$ and $\mathrm{CEF}^{\mathrm{r}} \mathrm{TP}^{\mathrm{r}}$, were determined using those antibiotics to which $492 \mathrm{c}$ is hypersensitive, i.e. carbenicillin, methicillin, flucloxacillin, mecillinam, cefuroxime, chloramphenicol, trimethoprim, nalidixic acid and novobiocin.

All CEF ${ }^{\mathrm{T}} \mathrm{TP}^{\mathrm{r}}$ recombinants (three tested) were indistinguishable from PAO8 on the basis of MICs to these antibiotics. The $\mathrm{CEF}^{\mathrm{s}} \mathrm{TP}^{\mathrm{s}}$ group (eight tested) could be subdivided on the basis of their trimethoprim, nalidixic acid and novobiocin MICs. Three recombinants were very sensitive to these antibiotics with trimethoprim and novobiocin MICs of $10 \mu \mathrm{g} \mathrm{ml}^{-1}$, and a nalidixic acid MIC of $5 \mu \mathrm{g} \mathrm{m} l^{-1}$. The other $C E F^{s} \mathrm{TP}^{\mathrm{s}}$ recombinants were moderately sensitive with the following MICs: trimethoprim $60 \mu \mathrm{g} \mathrm{ml}^{-1}$, novobiocin $50 \mu \mathrm{g} \mathrm{ml}^{-1}$, nalidixic acid $20 \mu \mathrm{g} \mathrm{ml}^{-1}$. All eight recombinants were equally sensitive to the $\beta$-lactam antibiotics and chloramphenicol, and with the exception of cefuroxime were as sensitive as $492 \mathrm{c}$. The third class of recombinant, $\mathrm{CEF}^{\mathrm{r}} \mathrm{TP}^{\mathrm{s}}$ (five tested), displayed moderate sensitivity to trimethoprim, nalidixic acid and novobiocin, with MICs of $60 \mu \mathrm{g} \mathrm{ml}^{-1}, 20 \mu \mathrm{g} \mathrm{ml}^{-1}$ and $100 \mu \mathrm{g} \mathrm{ml}^{-1}$, respectively.

From these results, we postulated that the antibiotic hypersensitive phenotype of $492 \mathrm{c}$ is determined by two separate genes, both linked to met-28 and ilv-202, when transferred into PAO8. We propose to call these genes $b l s A l$, coding for hypersensitivity to certain $\beta$-lactams and a moderate sensitivity to trimethoprim, nalidixic acid and novobiocin; and $\operatorname{tps} A 1$ coding for increased sensitivity to the latter three antibiotics but not the $\beta$-lactams. Table 4 shows the relevant MIC values for PAO8 and three typical recombinant strains PAJ1 (blsAl tpsAl), PAJ2 (blsAl) and PAJ3 (tpsAl).

\section{Transfer of tpsAI from 492 a to PAO8}

As shown in Table 2, the trimethoprim MIC for 492a is fivefold less than for PAO8. In addition, the increased sensitivity of 492c to trimethoprim, compared with 492a (eightfold), is of the same order as the MIC difference between PAJl and PAJ3 (sixfold) (see Table 4). 


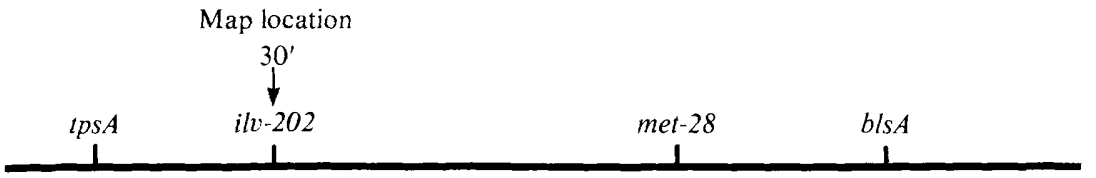

Fig. 2. Approximate locations of $b i s A$ and $\operatorname{tps} A$ on the basis of results obtained from plate matings between 492c Leu- (R68.45) and PAO8.

Table 4. Antibiotic sensitivities of PAO8 and typical recombinant strains PAJI, PAJ2 and PAJ3

\begin{tabular}{|c|c|c|c|c|c|}
\hline & & & MIC & & \\
\hline Antibiotic & Strain & PAO8 & $\begin{array}{c}\text { PAJ1 } \\
(b l s A l \text { tps } A l)\end{array}$ & $\begin{array}{c}\mathrm{PAJ} 2 \\
(\text { blsAI) }\end{array}$ & $\begin{array}{l}\mathrm{PAJ} 3 \\
(t p s A)\end{array}$ \\
\hline Carbenicillin & & 40 & 0.4 & $0 \cdot 4$ & 40 \\
\hline Methicillin & & 500 & 10 & 10 & 500 \\
\hline Flucloxacillin & & $>500$ & 50 & 50 & $>500$ \\
\hline Mecillinam & & 500 & 10 & 10 & 500 \\
\hline Cefuroxime & & 400 & 100 & 100 & 400 \\
\hline Chloramphenicol & & 40 & 20 & 20 & 40 \\
\hline Trimethoprim & & 200 & 10 & 60 & 60 \\
\hline Nalidixic acid & & 80 & 5 & 20 & 20 \\
\hline Novobiocin & & 500 & 10 & 50 & 10 \\
\hline
\end{tabular}

On this basis, we proposed that tpsAI is present in both $492 \mathrm{a}$ and $492 \mathrm{c}$, and that the antibiotic hypersensitivity of the latter strain can be explained by a mutation at the $b l s A$ locus, i.e. there is only a single gene difference between $492 \mathrm{a}$ and $492 \mathrm{c}$ accounting for antibiotic hypersensitivity.

Using the mapping procedure already described for $492 \mathrm{c}$, a donor derivative of $492 \mathrm{a}$ [ $492 \mathrm{a}$ $\mathrm{Leu}^{-}$(R68.45)] was plate mated with PAO8, and the $\mathrm{met}^{+}$and $i \mathrm{lv}^{+}$recombinants scored on DSTA containing either $100 \mu \mathrm{g}$ trimethoprim $\mathrm{ml}^{-1}$ or $100 \mu \mathrm{g}$ cefuroxime $\mathrm{ml}^{-1}$. As expected, a proportion of recombinants failed to grow well on trimethoprim, though all grew well on cefuroxime.

Mapping of bls $A$ and tps $A$ by transduction using phage F116L

From the plate mating results, it was possible to assign approximate chromosomal locations to bls $A I$ and tpsAI relative to $i l v-202$ and met-28 (see Fig. 2). However, to map these loci more precisely with respect to other markers in the $30 \mathrm{~min}$ region, transductional analysis was performed.

In the case of blsAl, a preparation of $F 116 \mathrm{~L}$ was grown on PAJ2 and used to transduce recipient strains carrying the auxotrophic markers met-28 (PAO8), ilv-202 (PAO8), proC130 (PAO969) and pyrB52 (PAO4). Selection was made for these markers and cotransduction of blsAl scored on DSTA plus $5 \mu \mathrm{g}$ carbenicillin $\mathrm{ml}^{-1}$.

In the case of tpsA1, transducing preparations of $F 116 \mathrm{~L}$ could not be propagated on any recombinants containing this marker, as they did not appear to support vegetative growth. However, tpsAl strains retained the receptors for this phage as they were good recipients in F116L-mediated transductions, so in order to map this locus, an ilv-202 tpsAl derivative, PAJ6, was constructed (via the intermediate strain PAJ5) and used as recipient in transductions mediated by F116L propagated on either PAO1, PAO2 (ser-3) or GMA 037 (hisV5037). In each case selection was made for $i l v^{+}$transductants and these were scored for co-inheritance of $t p s \mathrm{~A}^{+}$ and where appropriate, the unselected auxotrophic markers. Figure 3 shows the locations obtained for $b l s A$ and $\operatorname{tps} A$.

\section{Isolation of a blsA mutant of PAO969}

Working on the hypothesis that $492 \mathrm{c}$ could have arisen from 492a by a single step mutation at the $b l s A$ locus, we postulated that a similar $b l s A$ mutant could be isolated from a PAO strain. 


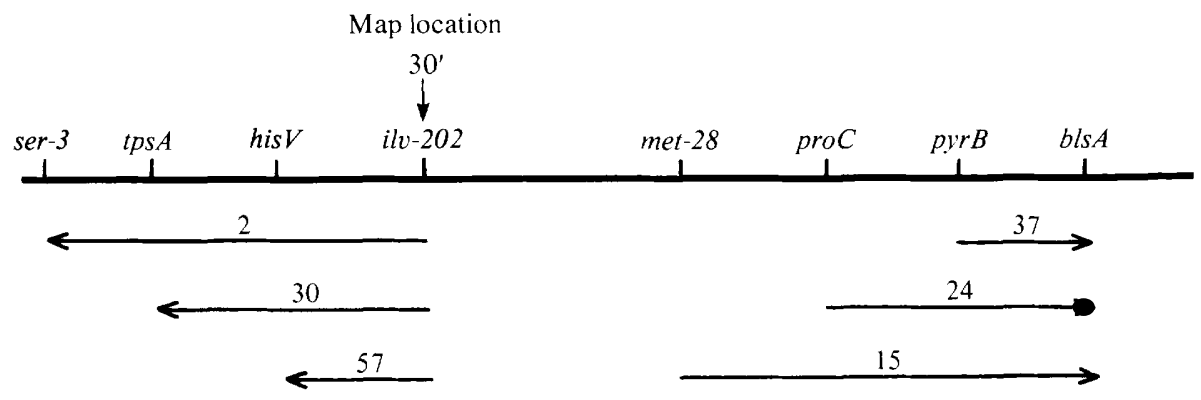

Fig. 3. Mapping of $t p s A l$ and $b l s A l$ by transduction. Values indicate percentage cotransduction using phage F116L (the mean of several experiments scoring at least 200 transductants). Arrowheads point to the unselected marker

Table 5. Results of F116L-mediated transductions to determine the relationship between nalB and $b l s A$

\begin{tabular}{|c|c|c|c|c|}
\hline \multirow[b]{2}{*}{ F116L grown on: } & \multirow[b]{2}{*}{ Recipient } & \multicolumn{3}{|c|}{$\begin{array}{l}\text { Percentage cotransduction } \\
\text { with proc } C^{+*}\end{array}$} \\
\hline & & bls $A 2$ & nalB4 & Wild-type \\
\hline $\mathrm{PAOl}\left(\mathrm{nalB}^{+}\right.$bls $\left.A 2^{+}\right)$ & PAO651। (blsA2) & - & - & 33 \\
\hline & PAO6524 (nalB4) & - & - & 43 \\
\hline PAO6002 (nalB4) & PAO6511 (blsA2) & - & 27 & $<0.5$ \\
\hline PAO6526 (blsA2) & PAO6524 (nalB4) & 19 & - & 3 \\
\hline
\end{tabular}

* In each case, selection was made for proC ${ }^{+}$and 200 transductants were scored for the co-inheritance of nalB4 $4^{+-}$and $b l s A 2^{+--}$on DSTA plus $50 \mu \mathrm{g}$ and $5 \mu \mathrm{g}$ carbenicillin $\mathrm{ml}^{-1}$, respectively.

PAO969 was mutagenized with EMS and the potential mutant colonies screened for hypersensitivity to carbenicillin by replica plating to DSTA plates containing $5 \mu \mathrm{g}$ carbenicillin $\mathrm{ml}^{-1}$. From approximately 5000 colonies screened, one failed to grow on medium containing carbenicillin. Fortuitously, this mutant (PAO6511) exhibited a similar antibiogram to the blsAI recombinants, i.e. PAO6511 was highly sensitive to carbenicillin, moderately sensitive to cefuroxime, trimethoprim, nalidixic acid, novobiocin and chloramphenicol, but remained resistant to cefoxitin, cephaloridine and benzyl penicillin. However, PAO6511 was more resistant to methicillin, mecillinam and flucloxacillin than a blsAl transductant of PAO969.

F116L transductions revealed $33 \%$ cotransduction between proC and the mutant locus. Furthermore, when F116L propagated on PAJ2 was used to transduce PAO6511, selecting for proC $^{+},<0.5 \%$ of the transductants were able to grow on $5 \mu \mathrm{g}$ carbenicillin $\mathrm{ml}^{-1}$, indicating a very close linkage between $b l s A l$ and the PAO mutation, which, on this basis, we have designated blsA2.

\section{Relationship between bls $A$ and nalB}

Rella \& Haas (1982) have described a class of mutants (nalB) in strain PAO which are resistant to nalidixic acid, novobiocin, carbenicillin and various other $\beta$-lactams. We examined two nalB mutants PAO6002 (nalB4) and PAO6006 (nalB9) to determine whether they showed increased resistance to cefuroxime, trimethoprim, methicillin and chloramphenicol. Both strains showed increased resistance to all four antibiotics with the following relative MICs with respect to PAO969: cefuroxime, $\times 3$; trimethoprim, $\times 6$; methicillin, $\times 5$; chloramphenicol, $\times 10$.

nalB has been mapped at $32 \mathrm{~min}$ on the PAO chromosome, distal to $p y r B$ using G101 transduction (Rella \& Haas, 1982), so we determined the location of $b l s A 2$ with respect to this locus, using strains PAO6511 and PAO6002. In order to perform reciprocal transductions, a proC derivative of PAO6002 (PAO6524) and a proc ${ }^{+}$derivative of PAO6511 (PAO6526) were 
constructed using F116c. Reciprocal transductions using F116L were performed between the $b l s A$ and nalB strains, in each case selecting for Pro ${ }^{+}$transductants and scoring for $b l s A$ and nalB on DSTA plates containing $5 \mu \mathrm{g}$ and $50 \mu \mathrm{g}$ carbenicillin $\mathrm{ml}^{-1}$. The results obtained are shown in Table 5, and suggest that $b l s A$ and nalB are separate, but closely linked loci, the gene order being proC-nalB-blsA.

\section{DISCUSSION}

Previous genetic studies on the intrinsic resistance of $P$. aeruginosa strain PAO have identified various chromosomal loci associated with this characteristic. Thus we tested the hypothesis that the loss of intrinsic resistance in $492 \mathrm{c}$ was due to a chromosomal mutation, rather than loss of a plasmid as suggested by May \& Ingold (1972). Inter-strain crosses between 492c Leu ${ }^{-}$(R68.45) and PAO8 identified two distinct loci, in the $30 \mathrm{~min}$ region of the chromosome, associated with antibiotic sensitivity. The first of these, $b l s A$, is associated with sensitivity to the $\beta$-lactams carbenicillin, methicillin, flucloxacillin, mecillinam and cefuroxime, and also the non- $\beta$-lactams chloramphenicol, trimethoprim, nalidixic acid and novobiocin and cannot be transferred to PAO from 492a.

The isolation of a $b l s A$ derivative of PAO following EMS mutagenesis provides further evidence that $492 \mathrm{c}$ could have arisen from 492a in vivo following a single-step mutation which was then selected out in the lung environment. This mutation-selection mechanism is also responsible for the emergence of mucoid strains of $P$. aeruginosa during the course of chronic pulmonary infections in CF patients. All strains of $P$. aeruginosa have the necessary structural genes to synthesize alginate, but these are normally subject to repression. Mutation in a regulator gene close to the major FP2 origin results in derepression of alginate synthesis giving rise to a mucoid strain (Govan \& Fyfe, 1978; Fyfe \& Govan, 1980; J. A. M. Fyfe \& J. R. W. Govan, unpublished).

Three major questions remain to be answered regarding antibiotic hypersensitivity associated with respiratory isolates. Firstly, whether all such isolates have the same mutation as $492 \mathrm{c}$ or whether they form a genetically heterogeneous group. We have examined several pairs of strains (i.e. hypersensitive and 'normal' from the same sputum specimen) isolated from different patients on the basis of antibiotic sensitivities and in general, the antibiograms have been similar to 492a and 492c (results not shown). Genetic analysis of one other hypersensitive strain gave identical results to those obtained with $492 \mathrm{c}$, even though this strain was isolated from a different patient and was of a different pyocin type. However, further work is required to determine whether the $b l s A$ locus is the only one involved in clinically associated antibiotic hypersensitivity.

Secondly, we would like to know the structural basis of this characteristic. We have previously reported that $492 \mathrm{c}$ has two extra outer membrane proteins when compared with 492a (Irvin et al., 1981), but further studies have failed to confirm that these are associated with antibiotic sensitivity.

Several studies on the structural basis of antibiotic hypersensitivity in $P$. aeruginosa have been reported (Noguchi et al., 1980; Curtis et al., 1981; Angus et al., 1982; Zimmerman, 1980; Kropinski et al., 1982; Darveau et al., 1983) and these have suggested that increased permeability of the cell envelope is responsible for this characteristic. In the case of $799 / 61$, a 'supersusceptible' mutant of strain 799 (Zimmerman, 1979), alterations in the LPS composition have been detected (Kropinski et al., 1982; Darveau et al., 1983). Similar mutants (envA) have been reported in Escherichia coli (Grundstrom et al., 1980; Boman et al., 1974).

The close linkage of $b l s A$ and $n a l B$ on the $P$. aeruginosa chromosome is interesting in view of the fact that nal $B$ mutants are more resistant to those antibiotics to which $b l s A$ strains are hypersensitive, and it is tempting to speculate that these two loci form part of a gene cluster controlling outer membrane permeability in this organism.

Finally, the clinical and biological significance of antibiotic hypersensitivity remains to be elucidated. Superficially it appears paradoxical that hypersensitive strains arise in patients undergoing aggressive antibiotic therapy, often with $\beta$-lactams. The lung, however, is a 
notoriously difficult site in which to achieve therapeutic levels of anti-pseudomonas antibiotics, and in this environment the vulnerability of hypersensitive strains may be more apparent than real.

Mutations to increased antibiotic sensitivity are also encountered amongst naturally occurring gonococci (Eisenstein \& Sparling, 1978; Lysko \& Morse, 1981) and it has been suggested that mucosal surface pathogens may find it advantageous to be flexible with respect to outer membrane structure and that increased permeability may aid colonization in this environment (Lysko \& Morse, 1981).

The emergence of antibiotic-hypersensitive strains of $P$. aeruginosa during the course of chronic pulmonary infections, regardless of the selective pressure, provides yet another example of this organism's ability to adapt to a changing environment.

This work was supported by the Scottish Hospital Endowments Research Trust and the Cystic Fibrosis Research Trust. The authors wish to thank Dr Dieter Haas for useful discussions and for supplying strains.

\section{REFERENCES}

Angus, B. L., Carey, A. M., Caron, D. A., Kropinski, A. M. B. \& HanCoCk, R. E. W. (1982). Outer membrane permeability in Pseudomonas aeruginosa: comparison of a wild-type with an antibioticsupersusceptible mutant. Antimicrobial Agents and Chemotherapy 21, 299-309.

Berche, P., Descamps, P., Avril, J. L., DaoulasLebourdelles, F. \& Veron, M. (1979). Effects of antibiotics on mucoid strains of Pseudomonas aeruginosa. Annales de Microbiologie 130A, 315-330.

Boman, H. G., Nordstrom, K. \& Normark, S. (1974). Penicillin resistance in Escherichia coli K 12: synergism between penicillinases and a barrier in the outer part of the envelope. Annals of the New York Academy of Sciences 235, 569-586.

Curtis, N. A. C., Orr, D., Boulton, M. G. \& Ross, G. W. (1981). Penicillin-binding proteins of Pseudomonas aeruginosa. Comparison of two strains differing in their resistance to $\beta$-lactam antibiotics. Journal of Antimicrobial Chemotherapy 7, 127-136.

Darveau, R. P. \& Hancock, R. E. W. (1983). Procedure for isolation of bacterial lipopolysaccharides from both rough and smooth Pseudomonas aeruginosa and Salmonella typhimurium strains. Journal of Bacteriology 155, 831-838.

Eisenstein, B. I. \& SParling, P. F. (1978). Mutations to increased antibiotic sensitivity in naturallyoccurring gonococci. Nature, London 271, 242-244.

Fyfe, J. A. M. \& Govan, J. R. W. (1980). Alginate synthesis in mucoid Pseudomonas aeruginosa: a chromosomal locus involved in control. Journal of General Microbiology 119, 443-450.

Fyfe, J. A. M. \& Govan, J. R. W. (1983). Genetic analysis of antibiotic hypersensitivity in Pseudomonas aeruginosa. Society for General Microbiology Quarterly 9, part 4, M10.

Govan, J. R. W. \& FyFe, J. A. M. (1978). Mucoid Pseudomonas aeruginosa and cystic fibrosis: resistance of the mucoid form to carbenicillin, flucloxacillin and tobramycin and the isolation of mucoid variants in vitro. Journal of Antimicrobial Chemotherapy 4, 233-240.
GraY, G. L. \& VASIL, M. L. (1981a). Isolation and genetic characterization of toxin deficient mutants of Pseudomonas aeruginosa PAO. Journal of Bacteriology 147, 275-281.

Gray, G. L. \& VASIL, M. L. (1981b). Mapping of a gene controlling the production of phospholipase $\mathrm{C}$ and alkaline phosphatase in Pseudomonas aeruginosa. Molecular and General Genetics 183, 403405 .

Grundstrom, T., Normark, S. \& Magnusson, K. E. (1980). Over-production of outer membrane protein suppresses env $A$-induced hyperpermeability. Journal of Bacteriology 144, 884-890.

HAAS, D. \& Holloway, B. W. (1976). R factor variants with enhanced sex factor activity in Pseudomonas aeruginosa. Molecular and General Genetics 144, 243251 .

HanNe, L. F., Howe, T. R. \& Iglewski, B. H. (1983). Locus of the Pseudomonas aeruginosa toxin A gene. Journal of Bacteriology 154, 383-386.

Holloway, B. W. (1969). Genetics of Pseudomonas. Bacteriological Reviews 33, 419-443.

Holloway, B. W. \& Crockett, R. J. (1982). Pseudomonas aeruginosa. Genetic Maps 2, 165-167.

Irvin, R. T., Govan, J. R. W., Fyfe, J. A. M. \& Costerton, J. W. (1981). Heterogeneity of antibiotic resistance in mucoid isolates of Pseudomonas aeruginosa obtained from cystic fibrosis patients: role of outer membrane proteins. Antimicrobial Agents and Chemotherapy 19, 1056-1063.

ISAAC, J. H. \& HollowaY, B. W. (1968). Control of pyrimidine biosynthesis in Pseudomonas aeruginosa. Journal of Bacteriology 96, 1732-1741.

Knudsen, E. T., Rolinson, G. N. \& Sutherland, R. (1967). Carbenicillin: a new semi-synthetic penicillin active against Pseudomonas pyocyanea. British Medical Journal 3, 75-78.

KRISHNAPILLAI, V. (1971). A novel transducing phage: its role in recognition of a possible new host controlled modification system in Pseudomonas aeruginosa. Molecular and General Genetics 114, 134 143. 
Kropinski, A. M., Kuzio, J., Angus, B. L. \& HANCOCK, R. E. W. (1982). Chemical and chromatographic analysis of lipopolysaccharide from an antibiotic-supersusceptible mutant of Pseudomonas aeruginosa. Antimicrobial Agents and Chemotherapy 21, 310-319.

Lysko, P. G. \& Morse, S. A. (1981). Neisseria gonorrhoeae cell envelope: permeability to hydrophobic molecules. Journal of Bacteriology 145, 946952.

Marks, M. I., Prentice, R., Swarson, R., Cotton, E. K. \& EICKHOFF, T. C. (1971). Carbenicillin and gentamicin: pharmacologic studies in patients with cystic fibrosis and pseudomonas pulmonary infections. Journal of Pediatrics 79, 822-828.

Matsumoto, H. \& Terawaki, Y. (1982). Chromosomal location of the genes participating in the formation of $\beta$-lactamase in Pseudomonas aeruginosa. In Drug Resistance in Bacteria, pp. 207--211. Edited by S. Mitsuhashi. Tokyo: Japan Scientific Societies Press.

MAY, J. R. \& INGOLD, A. (1972). Sensitivity of respiratory strains of Pseudomonas aeruginosa to carbenicillin. Journal of Medical Microbiology 6, 7782.

MEE, B. J. \& LEE, B. T. O. (1967). An analysis of histidine requiring mutants in Pseudomonas aeruginosa. Genetics, 55, 709-722.

Mills, B. J. \& Holloway, B. W. (1976). Mutants of Pseudomonas aeruginosa that show specific hypersensitivity to aminoglycosides. Antimicrobial Agents and Chemotherapy 10, 411-416.

Noguchi, H., Fukasawa, M., Komatsu, T., Iyobe, S. \& Mitsuhashi, S. (1980). Isolation of two types of Pseudomonas aeruginosa mutants highly sensitive to a specific group of $\beta$-lactam antibiotics and with defect in penicillin binding proteins. Journal of Antibiotics 33, 1521-1526.

Oxil, M., Iyobe, S. \& Mitsuhashi, S. (1983). Mapping of the gene specifying aminoglycoside 3 -phospho- transferase II on the Pseudomonas aeruginosa chromosome. Journal of Bacteriology 155, 643-649.

Rella, M. \& HaAs, D. (1982). Resistance of Pseudomonas aeruginosa PAO to nalidixic acid and low levels of $\beta$-lactam antibiotics: mapping of chromosomal genes. Antimicrobial Agents and Chemotherapy 22, 242-249.

Rolfe, B. \& Holloway, B. W. (1966). Alterations in host specificity of bacterial deoxyribonucleic acid after an increase in growth temperature of Pseudomonas aeruginosa. Journal of Bacteriology 92, 43-48.

Sinclair, M. I. \& Holloway, B. W. (1982). A chromosomally located transposon in Pseudomonas aeruginosa. Journal of Bacteriology 151, 569-579.

Stanisich, V. \& Holloway, B. W. (1972). A mutant sex factor of Pseudomonas aeruginosa. Genetical Research 19, 91-108.

Watson, J. M. \& Holloway, B. W. (1976). Suppressor mutations in Pseudomonas aeruginosa. Journal of Bacteriology 125, 780-786.

Watson, J. M. \& Holloway, B. W. (1978). Linkage map of Pseudomonas aeruginosa PAT. Journal of Bacteriology 136, 507-521.

Wretlind, B., BJorklind, A., Karlsson, I. \& HagelberG, A. (1983). Genetic mapping and characterization of a proelastase producing mutant of Pseudomonas aeruginosa. Abstracts of the $83 \mathrm{rd}$ Annual Meeting of the American Society for Microbiology, p. 37. Washington, D.C.: American Society for Microbiology.

Zimmerman, W. (1979). Penetration through the Gram-negative cell wall: a codeterminant of the efficacy of beta-lactam antibiotics. International Journal of Clinical Pharmacology and Biopharmacy 17 , 131-134.

Zimmerman, W. (1980). Penetration of beta-lactam antibiotics into their target enzymes in Pseudomonas aeruginosa: comparison of a highly sensitive mutant with its parent strain. Antimicrobial Agents and Chemotherapy 18, 94-100. 